\section{Resolvins are potent analgesics for arthritic pain}

\author{
Zhen-Zhong $\mathrm{Xu}$ and Ru-Rong Ji \\ Sensory Plasticity Laboratory, Pain Research Center, Department of Anesthesiology, Brigham and \\ Women's Hospital and Harvard Medical School, Boston, MA, USA
}

\section{Correspondence \\ Ru-Rong Ji, Department of Anesthesiology, Brigham and Women's Hospital, 75 Francis Street, Medical Research Building, Room 604, Boston, MA 02115, USA. E-mail: \\ rrji@zeus.bwh.harvard.edu}

\section{Keywords}

Resolvin; RvD1 precursor; lipid mediators; inflammatory pain; arthritis; dorsal root ganglion; spinal cord; TNF- $\alpha$

\author{
Received \\ 25 February 2011 \\ Revised \\ 28 February 2011 \\ Accepted \\ 2 March 2011
}

Arthritis-associated inflammatory pain represents a serious medical problem worldwide. Current treatments for arthritic pain are not very effective and cause unwanted side effects. Resolvins, including the resolvin D and resolvin E series, are a family of novel endogenous lipid mediators derived from $\omega-3$ polyunsaturated fatty acids and display potent anti-inflammatory and pro-resolution actions in animal models of inflammation. Emerging evidence also points to a potent anti-hyperalgesic role of resolvins in animal models of inflammatory pain. The study by Lima-Garcia et al. in this issue of the BJP demonstrated that systemic treatment with 17(R)-hydroxy-docosahexaenoic acid (17(R)HDoHE), the precursor of resolvin D series and its product, aspirin-triggered resolvin D1 (AT-RvD1), at very low doses $\left(1 \mu \mathrm{g} \cdot \mathrm{kg}^{-1}\right)$, reduced inflammatory pain in an adjuvantinduced arthritis model. Particularly 17(R)HDoHE reduced joint stiffness but not paw and joint oedema. Given their potency and safety profile, resolvins may represent a new class of analgesics well suited to treating inflammatory pain associated with arthritis.

\title{
LINKED ARTICLE
}

This article is a commentary on Lima-Garcia et al., pp. 278-293 of this issue. To view this paper visit http://dx.doi.org/ 10.1111/j.1476-5381.2011.01345.x

\section{Abbreviations}

DHA, docosahexaenoic acid; DRG, dorsal root ganglion; EPA, eicosapentaenoic acid; RvD1, resolvin D1; RvE1, resolvin E1; 17(R)HDoHE, 17(R)-hydroxy-4Z,7Z,10Z,13Z,15E,17R,19Z-docosahexaenoic acid; AT-RvD1, aspirin-triggered resolvin D1

A growing body of evidence suggests that the resolution of acute inflammation involves active biochemical pathways producing pro-resolution mediators (Serhan et al., 2008). The mechanisms of pro-resolution mediators are quite different from those of anti-inflammatory therapeutic agentss, such as COX-2 inhibitors which are known to delay resolution (Gilroy et al., 1999). The resolvins, such as resolvin D1 (RvD1) and resolving E1 (RvE1), represent a new family of proresolution mediators that are biosynthesized from $\omega-3$ fatty acids such as docosahexaenoic acid (DHA) and eicosapentaenoic acid (EPA) (Serhan et al., 2002). Resolvins display remarkable potency in resolving inflammation-related diseases such as periodontal disease, asthma and retinopathy (Serhan et al., 2008).

New evidence also points to an anti-nociceptive role of resolvins in inflammatory pain (Bang et al. 2010; Xu et al., 2010). Inflammatory pain after tissue injury such as arthritis results from peripheral sensitization, sensitization of nociceptor terminals in inflamed tissues and nociceptor cell bodies in the dorsal root ganglion (DRG), induced by inflammatory mediators such as tumor necrosis factor-alpha (TNF- $\alpha$ ), interleukin-1beta (IL-1 $\beta$ ) and prostaglandin (Cheng and Ji, 2008). Inflammatory pain is also mediated by central sensitization, sensitization of spinal cord dorsal horn neurons, induced by the same inflammatory mediators (TNF- $\alpha$, IL-1 $\beta$ and prostaglandin) produced in the spinal cord (Samad et al., 2001; Kawasaki et al. 2008). Xu et al. recently reported that peripheral (intraplantar) or spinal (intrathecal) administration of RvE1 or RvD1, at very low doses (0.3-20 ng), potently reduced inflammatory pain behaviours in acute and persistent mouse models of inflammation. RvE1 also reduced neutrophil infiltration and pro-inflammatory cytokine levels in the inflamed hind paw. In this setting, RvE1 abolished TNF- $\alpha-$ induced and transient receptor potential (TRP)V1-mediated 
synaptic plasticity in the spinal cord (Xu et al., 2010). A subsequent study showed that intraplantar administration of RvD1 reduced inflammatory pain by inhibiting the activities of several TRP channels such as TRPA1, TRPV3 and TRPV4, but not TRPV1 (Bang et al., 2010). Intrathecal pre-treatment with RvD1 (20-40 ng) also elicited enduring prevention of postoperative pain, whereas post-treatment with RvD1 produced a transient reduction of postoperative pain (Huang et al., 2011).

In this study, Lima-Garcia et al. (Lima-Garcia, 2011) demonstrate that a precursor of the resolvin D series, 17(R)hydroxy-docosahexaenoic acid (17(R)HDoHE), and its leukocyte-derived product, aspirin-triggered resolvin D1 (ATRvD1), elicited potent anti-hyperalgesic effects in adjuvantinduced arthritis in rats. Unlike earlier recent studies using local administration via intraplantar or intrathecal route, Lima-Garcia et al., used systemic administration, i.e., i.p. injections. Both pre-treatment with 17(R)HDoHE $\left(1 \mu \mathrm{g} \cdot \mathrm{kg}^{-1}\right)$ and post-treatment with 17(R)HDoHE on day $3\left(1 \mu \mathrm{g} \cdot \mathrm{kg}^{-1}\right)$ and day $14\left(2 \mu \mathrm{g} \cdot \mathrm{kg}^{-1}\right)$ significantly attenuated adjuvantinduced mechanical hyperalgesia for more than $6 \mathrm{~h}$, although 17(R)HDoHE appeared to produce the best antihyperalgesic effect in the early phase of inflammation (day 3). AT-RvD1 was also able to attenuate mechanical hyperalgesia at a very low dose $\left(0.3 \mu \mathrm{g} \cdot \mathrm{kg}^{-1}\right)$. Repeated injections of 17(R)HDoHE and AT-RvD1 reduced mechanical hyperalgesia for many days. Notably, the authors compared the antinociceptive efficacy of 17(R)HDoHE with that of currently used analgesics, including indomethacin (COX inhibitor), morphine (opioid receptor agonist), gabapentin (antiepileptic) and dexamethasone (anti-inflammatory steroid) in the same adjuvant model. It is very striking that the effective dose of 17 (R)HDoHE was $~ 500$ times lower than equiactive doses of morphine and $\sim 5000$ times lower than other currently used drugs.

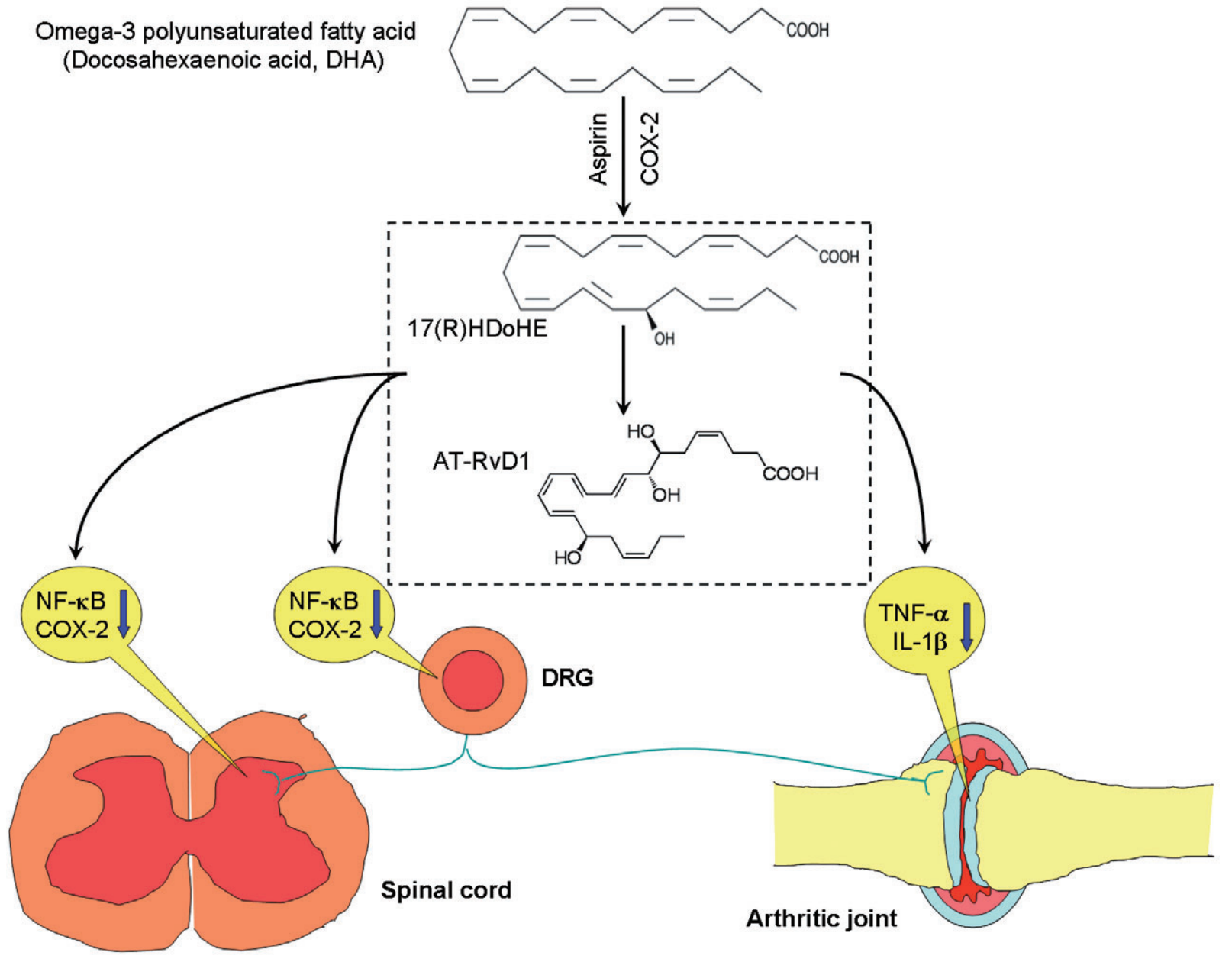

\section{Figure 1}

Possible pathways for the attenuation of arthritis-associated inflammatory pain by $17(\mathrm{R}) \mathrm{HDoHE}$ and its product aspirin-triggered resolvin D1 (AT-RvD1). 17(R)HDoHE is the precursor of the resolvin D series and is synthesized endogenously from the $\omega$ - 3 polyunsaturated fatty acid, docosahexaenoic acid (DHA), in the presence of aspirin-treated cyclooxygenase-2 (COX-2), to yield aspirin-triggered resolvin D1 (AT-RvD1). Adjuvant-induced hind paw inflammation results in increased levels of pro-inflammatory cytokines TNF- $\alpha$ and IL-1 $\beta$ in the inflamed paw, as well as increased expression of NK-KB or/and COX-2 in the dorsal root ganglion (DRG) and spinal cord. Up-regulation of these molecules has been implicated in the generation of inflammatory pain. 17(R)HDoHE and AT-RvD1 inhibit inflammatory pain by reducing the levels of TNF- $\alpha$ and IL-1 $\beta$ at the site of inflammation, and also inhibiting the expression of NK-кB or/and COX-2 in the DRG and spinal cord. 
Lima-Garcia et al. (2011) also investigated the underlying anti-hyperalgesic mechanisms of 17(R)HDoHE and AT-RvD1, as illustrated in Figure 1. First, repeated injections of 17(R)HDoHE and AT-RvD1 substantially reduced TNF- $\alpha$ and IL-1 $\beta$ levels in inflamed paw tissue. Second, a single treatment with 17(R)HDoHE inhibited the increase of NF- $\kappa$ B and COX-2 in the spinal cord and DRG. All these mechanisms have been strongly implicated in the pathogenesis of inflammatory pain via peripheral and central sensitization (Samad et al., 2001; Cheng and Ji, 2008; Kawasaki et al. 2008). The authors did not find significant increases in TNF- $\alpha$ and IL-1 $\beta$ in DRG and spinal cord tissues, after adjuvant-induced inflammation. However, the importance of these cytokines in regulating central sensitization and spinal cord-mediated hyperalgesia should not be underestimated. TNF- $\alpha$ and IL-1 $\beta$ may increase at different times after inflammation (Samad et al., 2001). Second, these cytokines could be released into the cerebrospinal fluid and thus would not be present in spinal cord tissue. Third, the receptors for these cytokines could be up-regulated after inflammation. Indeed, TNF- $\alpha$ and IL-1 $\beta$ can powerfully and rapidly regulate synaptic transmission in the spinal cord (Kawasaki et al., 2008). 17(R)HDoHE and AT-RvD1 may not only reduce the synthesis of TNF- $\alpha$ and IL- $1 \beta$, but also inhibit TNF- $\alpha$ and IL-1 $\beta$-induced enhancement of synaptic transmission, as RvE1 does (Xu et al., 2010).

An important finding in the paper by Lima-Garcia et al. is that repeated treatment with 17(R)HDoHE for 6 days reduced arthritic pain and joint stiffness, but not paw and joint oedema. Because pain is the major symptom in patients with arthritis, future studies on arthritis should be focused on pain reduction. In support of this, a recent study showed that TNF- $\alpha$ neutralization rapidly inhibited pain responses in the CNS before directly affecting joint inflammation in patients with rheumatoid arthritis (Hess et al., 2011). Indeed, after anti-TNF- $\alpha$ treatment, arthritic pain was reduced within $24 \mathrm{~h}$, whereas signs of joint inflammation, such as swollen joint counts, were reduced after 14 days (Hess et al., 2011). It is likely that continuous treatment with 17(R)HDoHE for 2 weeks would reduce joint oedema, by decreasing TNF- $\alpha$ levels.

It is interesting to note that 17(R)HDoHE and (AT-RvD1), as well as RvD1, are very effective in inhibiting mechanical hyperalgesia, but not heat hyperalgesia, after inflammation. In contrast, RvE1 is highly efficacious in reducing heat hyperalgesia (Xu et al., 2010). Thus, different resolvins may regulate different modalities of pain by targeting different pain receptors such as thermal and mechanical receptors (using different TRP channels). AT-RvD1 and RvD1 activate the same receptors, GPR32 (human) and ALX/FPR2 (murine), two recently identified GPCRs (Krishnamoorthy et al., 2010). Whether or not 17-HDoHE also acts at these GPCRs remains to be established. In addition to AT-RD1, 17(R)-HDoHE is also converted to other aspirin-triggered D series resolvins, including AT-RvD2, AT-RvD3, AT-RvD4 and AT-RvD5 (Serhan et al., 2002; Serhan, 2007). The analgesic actions of the other D-series resolvins need further investigation.

Inflammatory pain, such as arthritic pain, is a growing health problem worldwide. Rheumatoid arthritis alone affects up to $1 \%$ of the population, and pain is the initial and prevailing symptom of this condition. Current treatments for inflammatory pain are limited by side effects. For example, acute opioid treatment produces respiratory depression, sedation, nausea, vomiting and constipation, whereas chronic opioid treatment may cause addiction. Long-term treatment with COX-2 inhibitors produces unwanted cardiovascular defects. Anti-TNF- $\alpha$ treatment may also cause problems with infection by suppressing the immune system (Sommer and Birklein, 2010; Xu et al. 2010). Given the remarkable antihyperalgesic efficacy of the resolvins and the safety profiles associated with these endogenous lipid mediators and dietary supplements, resolvins and their metabolically stable analogues may represent a novel class of analgesics for the management of inflammation-associated pain such as arthritic pain, low back pain, inflammatory bowel syndrome and postoperative pain.

\section{Acknowledgements}

We thank Dr Charles N. Serhan for valuable discussion. Our work was supported in part by the NIH R01 grant NS67686.

\section{Conflicts of interest}

The authors declare they have no conflicts of interest.

\section{References}

Bang S, Yoo S, Yang TJ, Cho H, Kim YG, Hwang SW (2010). Resolvin D1 attenuates activation of sensory transient receptor potential channels leading to multiple anti-nociception. Br J Pharmacol 161: 707-720.

Cheng JK, Ji RR (2008). Intracellular signaling in primary sensory neurons and persistent pain. Neurochem Res 33: 1970-1978.

Gilroy DW, Colville-Nash PR, Willis D, Chivers J, Paul-Clark MJ, Willoughby DA (1999). Inducible cyclooxygenase may have anti-inflammatory properties. Nat Med 5: 698-701.

Hess A, Axmann R, Rech J, Finzel S, Heindl C, Kreitz S et al. (2011). Blockade of TNF- rapidly inhibits pain responses in the central nervous system. Proc Natl Acad Sci U S A 108: 3731-3736.

Huang L, Wang CF, Serhan CN, Strichartz G (2011). Enduring prevention and transient reduction of postoperative pain by intrathecal resolvin D1. Pain 152: 557-565.

Kawasaki Y, Zhang L, Cheng JK, Ji RR (2008). Cytokine mechanisms of central sensitization: distinct and overlapping role of interleukin-1beta, interleukin-6, and tumor necrosis factor-alpha in regulating synaptic and neuronal activity in the superficial spinal cord. J Neurosci 28: 5189-5194.

Krishnamoorthy S, Recchiuti A, Chiang N, Yacoubian S, Lee CH, Yang R et al. (2010). Resolvin D1 binds human phagocytes with evidence for proresolving receptors. Proc Natl Acad Sci U S A 107: $1660-1665$.

Lima-Garcia JF, Dutra RC, da Silva KABS, Motta EM, Campos MM, Calixto JB (2011). The precursor of resolvin D series and aspirin-triggered resolvin D1 display anti-hyperalgesic properties in adjuvant-induced arthritis in rats. Br J Pharmacol 164: 278-293. 
Samad TA, Moore KA, Sapirstein A, Billet S, Allchorne A, Poole S et al. (2001). Interleukin-1beta-mediated induction of Cox-2 in the CNS contributes to inflammatory pain hypersensitivity. Nature 410: 471-475.

Serhan CN (2007). Resolution phase of inflammation: novel endogenous anti-inflammatory and proresolving lipid mediators and pathways. Annu Rev Immunol 25: 101-137.

Serhan CN, Hong S, Gronert K, Colgan SP, Devchand PR, Mirick G et al. (2002). Resolvins: a family of bioactive products of omega-3 fatty acid transformation circuits initiated by aspirin treatment that counter proinflammation signals. J Exp Med 196: $1025-1037$.

Serhan CN, Chiang N, Van Dyke TE (2008). Resolving inflammation: dual anti-inflammatory and pro-resolution lipid mediators. Nat Rev Immunol 8: 349-361.

Sommer C, Birklein F (2010). Fighting off pain with resolvins. Nat Med 16: 518-520.

Xu ZZ, Zhang L, Liu T, Park JY, Berta T, Yang R et al. (2010). Resolvins RvE1 and RvD1 attenuate inflammatory pain via central and peripheral actions. Nat Med 16: 592-597. 